\title{
V-like Formations in Flocks of Artificial Birds
}

\author{
Andre Nathan \\ Valmir C. Barbosa* \\ Universidade Federal do Rio de Janeiro \\ Programa de Engenharia de Sistemas e Computação, COPPE \\ Caixa Postal 68511 \\ 21941-972 Rio de Janeiro - RJ, Brazil
}

\begin{abstract}
We consider flocks of artificial birds and study the emergence of V-like formations during flight. We introduce a small set of fully distributed positioning rules to guide the birds' movements and demonstrate, by means of simulations, that they tend to lead to stabilization into several of the well-known V-like formations that have been observed in nature. We also provide quantitative indicators that we believe are closely related to achieving V-like formations, and study their behavior over a large set of independent simulations.
\end{abstract}

Keywords: Artificial birds, Boids, Flocking, V-like flight formations.

${ }^{*}$ Corresponding author (valmir@cos.ufrj.br). 


\section{Introduction}

Migratory birds in flight often group into V-like formations, that is, they tend to self-organize into a flight formation having very few individuals in lead positions while the others group behind them near the edges of imaginary trailing V's whose vertices are the lead birds. While it seems to be a consensus that group flight provides greater protection against predators, the reasons for the particular grouping into laterally slanted, nearly straight lines have elicited two distinct lines of explanation, following the seminal works in [12] and [5], respectively.

The first one has evoked the aerodynamics of bird flight, whose essence is that each flying individual creates an upwash region behind it, just off the tips of its wings, such that another individual benefits greatly (in terms of requiring less exertion during flight) if it places one of its wings in that region. For relatively large birds, which are the ones that have been observed to group into V-like formations most often, this seems to be the main reason for formation flight [8, 6, 2, 18, 21. The second, competing line of reasoning has been that flying in a somewhat skewed position relative to the bird in front is crucial for an individual's orientation, in addition to allowing unhindered visual communication and therefore helping avoid collisions. For relatively small birds, the aerodynamic benefits are less relevant and, moreover, the observed lateral and longitudinal separations between nearest birds seem to be correlated in a way that strongly supports this vision-related explanation 3 .

In this study, we consider simple artificial birds and address the question of whether positioning rules exist that, during flight, can lead a flock to settle into some stable V-like formation. Such rules should be founded on a blend of the basic explanatory trends of placing more weight on aerodynamics- or visionrelated justifications, thus consonant with the current belief that the traits that allow natural birds to take advantage of one or the other benefit evolved concomitantly [14. They should, in addition, be inherently distributed, so that only sensory information is to guide each bird's course of action, and also motivated by the birds' innate drive at flying as a flock. As we demonstrate in the remainder of this letter, rules with these characteristics do exist that are both robust (in the sense of ensuring that stable configurations can be expected to be reached) and flexible (allowing for a rich variety of V-like formations).

Our work is preceded by important related contributions, notably by the simple rules in [15], which in essence say that birds should avoid colliding with one another while attempting to maintain the same pace as the nearest birds as well as seeking to be positioned as near their center as possible. Although such rules are not meant to give rise to V-like formations (only to the flight as a group itself), we note that not even the further addition to them in [4] (strive to keep some portion of the visual field unobstructed) has been successful. We also mention the fuzzy rules introduced in [10, 11, but they too fail to induce V-like formations, except in the case of very special initial conditions. Less related to this study but very relevant nonetheless are mathematical analyses of flocking, 
both in the sense of [15] (cf., for example, [20, 13]) and under the assumption of a $\mathrm{V}$-formation (e.g., [19]).

\section{Positioning rules}

Our own set of rules is very succinct, comprising only three rules, each related to one of the three guiding principles discussed earlier (birds should flock, be afforded some unobstructed view in the direction of flight, and benefit from regions of upwash). They are the following.

Rule 1 (coalescing rule): Seek the proximity of the nearest bird.

Rule 2 (gap-seeking rule): If Rule 1 is no longer applicable, seek the nearest position that affords an unobstructed longitudinal view.

Rule 3 (stationing rule): Apply Rule 2 while the view that is sought is not obtained or the effort to keep up with the group decreases.

These three rules are an informal expression of our understanding of how each of the guiding principles is to influence a bird's actions. First of all, Rules 1 and 2 imply that we make a distinction between two modes of behavior, one succeeding, or sometimes alternating with, the other: birds are to seek first the closeness of the group, and only then being afforded some clear view. Secondly, Rules 2 and 3 imply that the sensory input that keeps the bird moving toward a relative position of clear view is not only visual but also originates in the ease with which it is keeping up with the group.

Rules 1-3 require further specification before we describe our computational experiments and their results. Note, first, that both Rule 1 and Rule 2 make implicit reference to the region of space where some entity is to be sought (the nearest bird in Rule 1, the nearest position with a clear view in Rule 2). We assume, in both cases, that this region is delimited, on the plane of movement and both to the right and left of the direction of movement, by an angle $\alpha / 2$. We assume also that $\alpha \leq 180^{\circ}$ (this is in spite of the fact that many birds in nature have visual fields delimited by larger angles, since $\alpha$ does not delimit a bird's visual field but the region where it is to seek the input needed by Rules 1 and 2).

In a similar vein, Rules 1 and 3 allude, also implicitly, to the existence of a closed region surrounding each bird inside which proximity is attained (Rule 1) or upwash is found (Rule 3). Given the aforementioned constraints on $\alpha$, and disregarding as negligible the upwash that some authors claim may be generated by a flying bird even in the space just ahead of it [19, 1], we assume that this region is as follows, with reference to Figure 1 [in this figure, as in others to come, an artificial bird is depicted as a filled circle (the bird's body) with two protruding straight-line segments (its wings)]. If $i$ is the bird seeking the proximity of another (say $j$ ) in Rule 1, then proximity is attained if the body of $i$ is in $U_{j}^{-} \cup D_{j} \cup U_{j}^{+}$. As for finding upwash in Rule 3, all that is needed is that any portion of bird $i$, body or a wing segment of any size, be in $U_{j}^{-} \cup U_{j}^{+}$while 


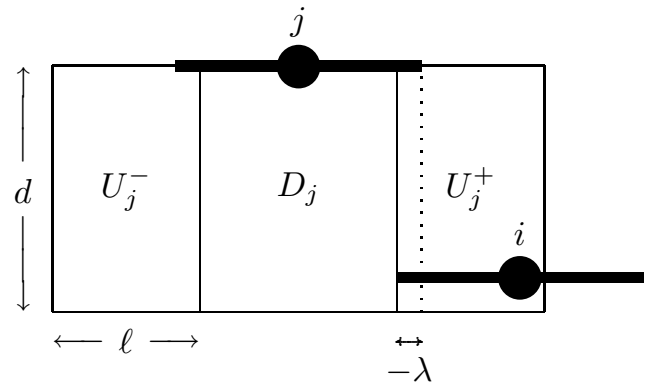

Figure 1: A flying bird $j$ 's upwash $\left(U_{j}^{-}\right.$and $\left.U_{j}^{+}\right)$and downwash $\left(D_{j}\right)$ regions. Bird $i$ is positioned for maximum upwash.

no portion is in $D_{j}$. In this case, the optimal relative placement of $i$ and $j$ (i.e., the relative placement for maximum upwash) occurs when the lateral separation between them is $\lambda=(\pi / 4-1) w / 2 \approx-0.1073 w$, where $w$ is a bird's wingspan, assumed the same for all birds [8, 9, 16, (the birds' longitudinal separation inside $U_{j}^{-} \cup U_{j}^{+}$is thought to be irrelevant for maximum upwash [12, 8, 16]). So, for optimality, $i$ and $j$ must have laterally overlapping wings.

\section{Computational results and discussion}

Our simulation algorithm operates on $n$ birds and runs for $T$ time steps from some initial positioning of the birds inside a unit square in two-dimensional space. We assume that the lateral and longitudinal directions are parallel to the square's sides. In order to move according to Rules $1-3$, a bird's position relative to the others' must undergo occasional lateral as well as longitudinal displacements. Except for the occurrence of these displacements, we assume that birds have no lateral speed and that they all fly at the same longitudinal speed. We then implement displacements by simply adding (or subtracting) a fixed amount $\Delta x$ (laterally) or $\Delta y$ (longitudinally) to a bird's position per time step. Birds are in no way constrained to flying inside the original unit square, even though by Rule 1 it seems reasonable to expect that a fixed-size square exists which, at all time steps, encompasses all birds. They may then be thought of as inhabiting unbounded two-dimensional space.

At each time step, a bird follows Rule 1, if applicable, by altering its position via $\pm \Delta x$ or $+\Delta y$. If Rule 1 is not applicable, then applying Rule 2 (and controlling its application via Rule 3) requires a precise criterion for seeking "the nearest position that affords an unobstructed longitudinal view." Let us call a gap, in the current time step, any lateral span that contains the lateral coordinate of no portion of any bird. A gap is maximal if it is contained in no other gap; maximal gaps, therefore, may be regarded as inducing longitudinal stripes of empty space, each stripe delimited by some bird's wing tip on at least one side. Our algorithm implements the criterion required by Rule 2 as follows. 
Let $i$ be the bird in question and consider all maximal gaps that are at least $w+2 \lambda$ wide (this includes the two outermost gaps, which are infinitely wide). The position that $i$ seeks is given by the nearest wing tip delimiting such a gap, provided the width- $(w+2 \lambda)$ extension of that wing into the gap can be seen by $i$ without obstruction. Aiming at that position, $\pm \Delta x$ is applied as needed, and eventually Rule 1 may once again become applicable. As for what remains unspecified of Rule 3, our algorithm implements the lateral separation by $\lambda$ directly (i.e., Rule 2 is applied if upwash has not been found or is not optimal).

Collisions are avoided at all time steps whenever attempting a displacement. Two birds are allowed to get as close to each other as having no lateral separation between them or a longitudinal separation of $\epsilon$. If the desired displacement infringes this, then it is not effected and the bird simply chooses randomly between a longitudinal displacement of $+\Delta y$ or $-\Delta y$, which is only applied if possible.

We have conducted extensive simulations for $n=15,25,35$ and $\alpha=170^{\circ}, 180^{\circ}$ (the occurrence of V-like formations in large flocks is inherently difficult and is rarely observed in nature [17, thence our moderate choices for the value of $n$ ). All simulations used $T=2000$ and the values listed in Table 1 for the distancerelated parameters. For a quantitative evaluation, we concentrate on the five indicators listed next, for which results were obtained as averages over 1000 independent simulations, each one starting at a random placement of the $n$ birds inside the unit square. A sampler of the formations achieved after $T$ time steps is shown in Figure 2, the averages for the five indicators in Figure 3 ,

Time for stabilization: Number of time steps until all birds stop moving relative to one another (taken as $T$ for the simulations in which this does not happen).

Number of lead birds: How many birds intersect no other's upwash regions.

Number of unconnected groups: A set of birds is counted as an unconnected group if no bird outside it intersects any of its own birds' upwash regions, and conversely [e.g., the situation in Figure 2(c) contains two groups].

Number of straight-line segments: A straight-line segment joins a trailing bird - one whose upwash regions no other bird intersects - to either a lead bird or a "bifurcation" bird - one whose upwash regions are intersected by two other birds (e.g., the situation in Figure 4 contains two straight-line segments).

Mean distance to nearest straight-line segments: Each of the $n$ birds contributes one distance to the mean, unless it is a lead or "bifurcation" bird, in which case it contributes the average distance to the two nearest segments.

Except for the time for stabilization, all indicators refer to the end of the simulation. 

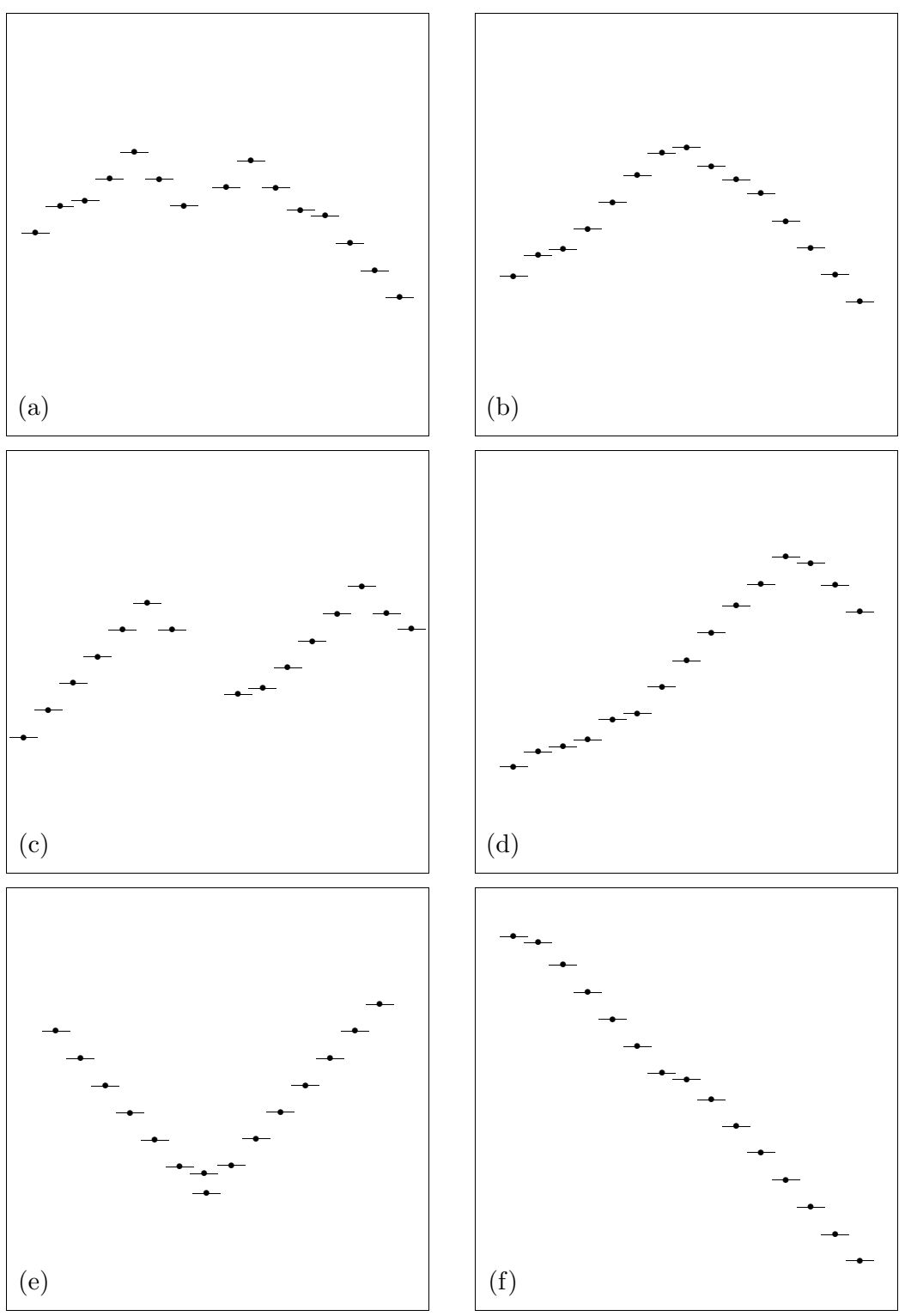

Figure 2: Sample formations after $T=2000$ time steps from independent, random initial placements for $n=15$, with $\alpha=170^{\circ}$ (a, c, e) and $\alpha=180^{\circ}$ (b, $\mathrm{d}, \mathrm{f})$. Samples include the celebrated $\mathrm{W}$ (a) and V (b) formations, in addition to a formation into multiple unconnected groups (c) and those formations that in [7] have been called the $\mathrm{J}(\mathrm{d})$, the inverted $\mathrm{V}$ (e), and the echelon (f). 

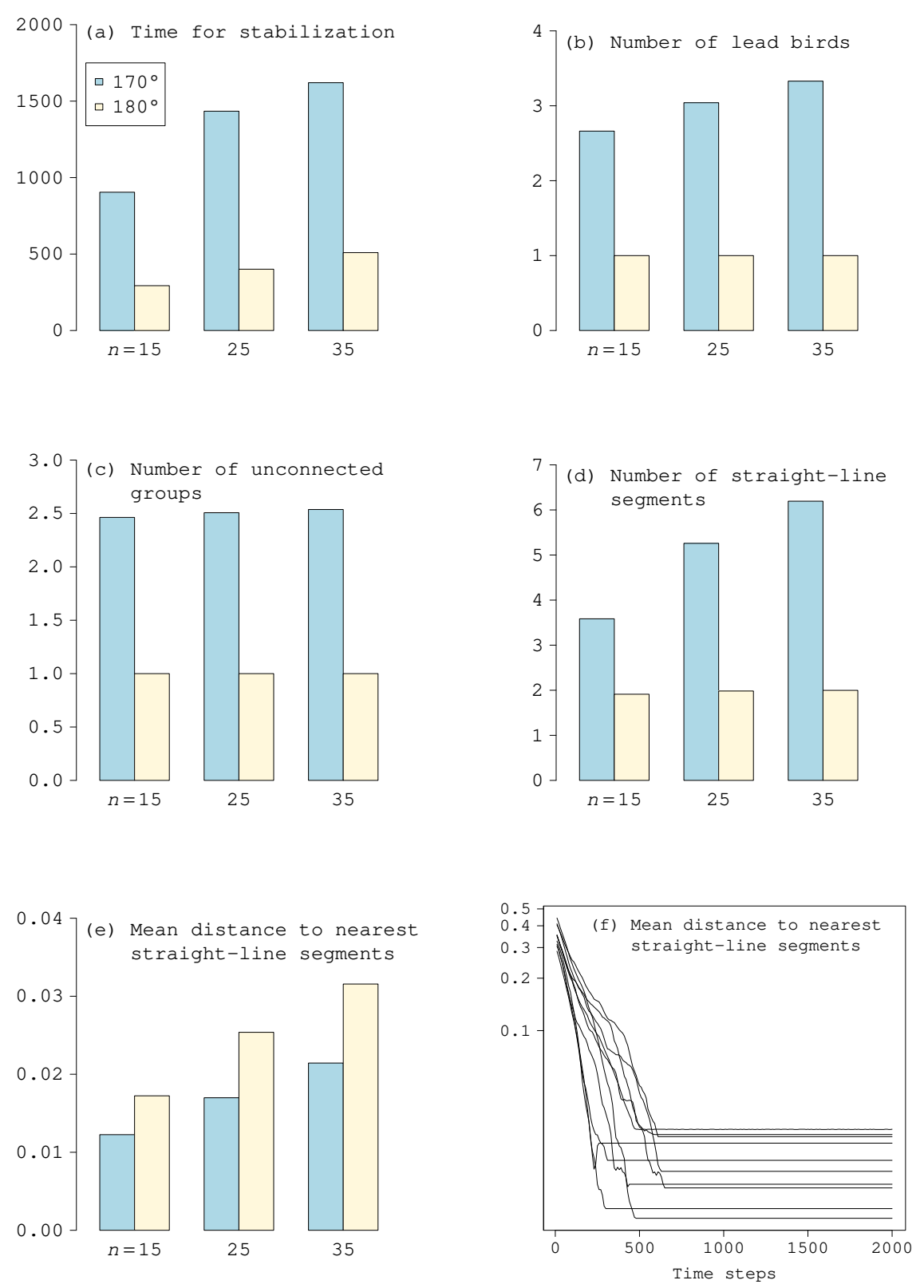

Figure 3: The five indicators, averaged over 1000 independent simulations (a-e), and the time evolution of the fifth indicator for ten randomly chosen simulations with $n=25$ and $\alpha=170^{\circ}$ (f). 
Table 1: Parameter values $(\times 1 / 768)$.

\begin{tabular}{ccl}
\hline Parameter & Value & Description \\
\hline$\ell$ & 30 & Lateral size of upwash region (cf. Figure 1) \\
$d$ & 50 & Longitudinal size of upwash region (cf. Figure 1) \\
$w$ & 50 & Wingspan \\
$\Delta x$ & 3 & Lateral displacement per time step \\
$\Delta y$ & 3 & Longitudinal displacement per time step \\
$\epsilon$ & 9 & Margin for longitudinal collision \\
\hline
\end{tabular}

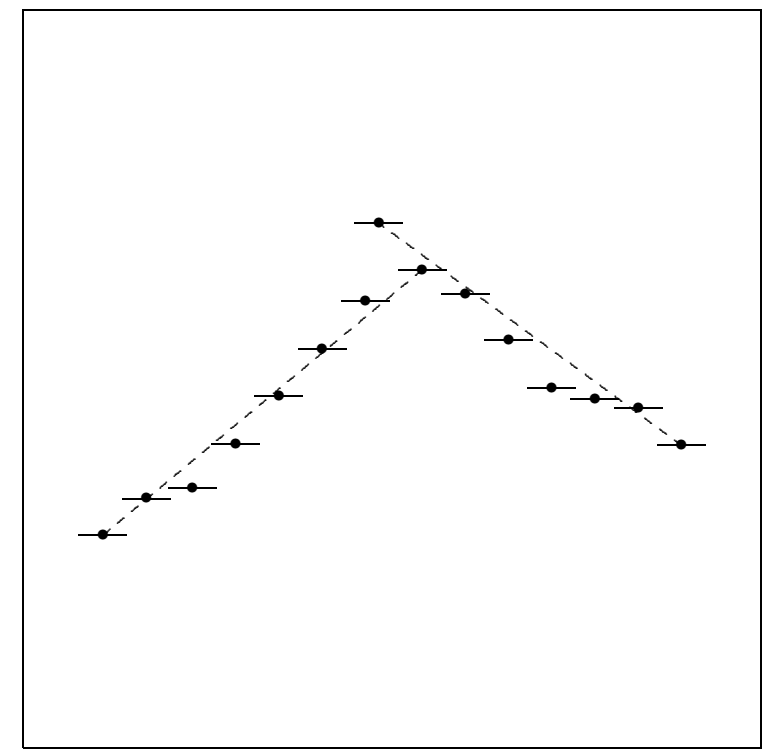

Figure 4: Flight formation with two straight-line segments (shown in dashes).

The plots in Figure 3 reveal that, for $\alpha=180^{\circ}$, the average formation into which the $n$ birds settle seems to become stable relatively quickly, and also to be evocative of the canonical $\mathrm{V}$ formation: one lead bird (hence one single group), and two straight-line segments. Decreasing $\alpha$ to $170^{\circ}$ delays stabilization significantly while allowing for an increased number of lead birds and of unconnected groups, and consequently of straight-line segments as well. Behind all these effects are the facts that, with the smaller angle, a bird tends to have a more limited set of choices for the nearest bird needed in Rule 1, and also fewer maximal gaps for use in the implementation of Rule 2. Upon reaching stability, however, the number of birds clustered around each straightline segment tends to be smaller, and thus so does the mean distance between the birds and the nearest segments.

We finalize by examining, in Figure 3(f), a closer glimpse into ten (out of the 1000) simulations for $n=25$ and $\alpha=170^{\circ}$. The figure indicates that, 
even though at this reduced angle stability is on average harder to achieve, there are simulations in which the mean distance to the nearest straight-line segments drops nearly vertiginously. This suggests, as in fact we have confirmed by examining all the data more closely, that the average time for stabilization is being influenced by some simulations that did not fully stabilize and contribute each $T=2000$ time steps to the average.

\section{Acknowledgments}

We acknowledge partial support from CNPq, CAPES, and a FAPERJ BBP grant.

\section{References}

[1] M. Anderson and J. Wallander. Kin selection and reciprocity in flight formation? Behavioral Ecology, 15:158-162, 2004.

[2] J. P. Badgerow. An analysis of function in the formation flight of Canada geese. The Auk, 105:749-755, 1988.

[3] C. J. Cutts and J. R. Speakman. Energy savings in formation flight of pink-footed geese. Journal of Experimental Biology, 189:251-261, 1994.

[4] G. W. Flake. The Computational Beauty of Nature: Computer Explorations of Fractals, Chaos, Complex Systems, and Adaptation. The MIT Press, Cambridge, MA, 1998.

[5] L. L. Gould and F. Heppner. The vee formation of Canada geese. The Auk, 91:494-506, 1974.

[6] F. R. Hainsworth. Precision and dynamics of positioning by Canada geese flying in formation. Journal of Experimental Biology, 128:445-462, 1987.

[7] F. Heppner. Avian flight formations. Bird-Banding, 45:160-169, 1974.

[8] D. Hummel. Aerodynamic aspects of formation flight in birds. Journal of Theoretical Biology, 104:321-347, 1983.

[9] D. Hummel. Formation flight as an energy-saving mechanism. Israel Journal of Zoology, 41:261-278, 1995.

[10] I. Lebar Bajec, N. Zimic, and M. Mraz. Fuzzifying the thoughts of animats. In Proceedings of the 10th International Fuzzy Systems Association World Congress, volume 2715 of Lecture Notes in Computer Science, pages 195202, 2003.

[11] I. Lebar Bajec, N. Zimic, and M. Mraz. Simulating flocks on the wing: the fuzzy approach. Journal of Theoretical Biology, 233:199-220, 2005. 
[12] P. B. S. Lissaman and C. A. Shollenberger. Formation flight of birds. Science, 168:1003-1005, 1970.

[13] R. Olfati-Saber. Flocking for multi-agent dynamic systems: algorithms and theory. IEEE Transactions on Automatic Control, 51:401-420, 2006.

[14] J. M. V. Rayner. Fat and formation in flight. Nature, 413:685-686, 2001.

[15] C. W. Reynolds. Flocks, herds, and schools: a distributed behavioral model. Computer Graphics, 21:25-34, 1987.

[16] P. Seiler, A. Pant, and K. Hedrick. Analysis of bird formations. In Proceedings of the 41st IEEE Conference on Decision and Control, volume 1, pages 118-123, 2002.

[17] P. Seiler, A. Pant, and K. Hedrick. A systems interpretation for observations of bird V-formations. Journal of Theoretical Biology, 221:279-287, 2003.

[18] J. R. Speakman and D. Banks. The function of flight formations in greylag geese Anser anser; energy saving or orientation? Ibis, 140:280-287, 1998.

[19] T. Sugimoto. A theoretical analysis of formation flight as a nonlinear selforganizing phenomenon. IMA Journal of Applied Mathematics, 68:441-470, 2003.

[20] J. Toner and Y. Tu. Flocks, herds, and schools: a quantitative theory of flocking. Physical Review E, 58:4828-4858, 1998.

[21] H. Weimerskirch, J. Martin, Y. Clerquin, P. Alexandre, and S. Jiraskova. Energy saving in flight formation. Nature, 413:697-698, 2001. 